\title{
Postura crânio-cervical em mulheres disfônicas
}

\section{Craniocervical posture in dysphonic women}

\author{
Delaine Rodrigues Bigaton ${ }^{1}$, Kelly Cristina Alves Silvério² ${ }^{2}$ Kelly Cristina dos Santos Berni ${ }^{3}$, Giovanna Distefano ${ }^{4}$, \\ Fabiana Forti ${ }^{5}$, Rinaldo Roberto de Jesus Guirro ${ }^{6}$
}

\begin{abstract}
RESUMO
Objetivo: Analisar a postura e a função da região crânio-cervical em sujeitos disfônicos. Métodos: Participaram do estudo 28 mulheres (31,25 $\pm 8,14$ anos), divididas em dois grupos: experimental ( $\mathrm{N}=16$ portadoras de disfonia) e controle ( $\mathrm{N}=12$ clinicamente normais). As voluntárias foram submetidas à avaliação do Índice de Disfunção Crânio-Cervical (IDCC) e fotogrametria, sendo determinado o ângulo anterior formado entre a sétima vértebra cervical e o tragus, o qual corresponde à posição da cabeça no plano sagital. A análise das fotos foi realizada por três examinadores, duas vezes cada um, com intervalo de uma semana entre elas. A análise dos dados constou do teste de Shapiro-Wilk, seguido do teste t de Student, $(\mathrm{p}<0,05)$. Resultados: Em relação à fotogrametria, não houve diferença $(\mathrm{p}=0,2565)$ entre os valores médios do ângulo anterior do grupo controle $(50,92 \pm 5,18$ graus) e do grupo experimental (49,63 $\pm 5,46$ graus). O IDCC mostrou que o grupo experimental apresentou disfunção crânio-cervical, sendo 37,5 \% leve, 37,5\% moderada e $25 \%$ severa. Já no grupo controle $100 \%$ das voluntárias apresentaram disfunção crânio-cervical leve. Conclusão: Não houve diferença na posição da cabeça entre os grupos avaliados. Porém, as mulheres disfônicas apresentaram disfunção crânio-cervical mais acentuada que as do grupo controle.
\end{abstract}

Descritores: Postura/fisiologia; Disfonia/complicações; Coluna vertebral; Cabeça; Distúrbios da voz; Fotogrametria

\section{INTRODUÇÃO}

A postura pode ser entendida como a posição do corpo ou de uma parte dele ou, ainda, o modo de mantê-lo ou de compor movimentos. Uma postura inadequada pode levar a dor e alteração funcional de vários sistemas ${ }^{(1)}$. Como exemplo, pode-se citar a protrusão da cabeça, a qual pode afetar a função dos músculos anteriores do pescoço, como esternocleidomastóideo, escaleno, supra e infra-hiódeos e, consequentemente, resultar em alterações na produção vocal.

Trabalho realizado no Programa de Pós-Graduação em Fisioterapia e nos Cursos de Graduação em Fisioterapia e Fonoaudiologia da Universidade Metodista de Piracicaba - UNIMEP - Piracicaba (SP), Brasil.

(1) Doutora, Professora do Programa de Pós-Graduação em Fisioterapia da Universidade Metodista de Piracicaba - UNIMEP - Piracicaba (SP), Brasil. (2) Doutora, Professora do Programa de Pós-Graduação em Distúrbios da Comunicação da Universidade Tuiuti do Paraná - UTP - Curitiba (PR), Brasil.

(3) Pós-Graduanda (Doutorado) em Biologia Buco-Dental da Faculdade de Odontologia da Universidade Estadual de Campinas - UNICAMP - Piracicaba (SP), Brasil.

(4) $\mathrm{PhD}$ student, Physical Medicine and Rehabilitation, University of Pittsburgh - UP - Pittsburgh (PA), USA.

(5) Doutora, Professora do Curso de Fisioterapia das Faculdades Integradas Einstein de Limeira - FIEL - Limeira (SP), Brasil.

(6) Doutor, Professor do Departamento de Biomecânica, Medicina e Reabilitação do Aparelho Locomotor da Faculdade de Medicina de Ribeirão Preto - USP - Ribeirão Preto (SP), Brasil.

Endereço para correspondência: Rinaldo Roberto de Jesus Guirro. Av. Bandeirantes, 3900, Monte Alegre, Ribeirão Preto (SP), Brasil, CEP: 14049900. E-mail: rguirro@ fmrp.usp.br

Recebido em: 7/7/2009; Aceito em: 8/3/2010
Alguns autores têm observado clinicamente que as disfonias, em especial as hipercinéticas, podem estar relacionadas às disfunções da região cervical, tais como: dor (no repouso ou durante a função), hiperatividade dos músculos que envolvem essa região ${ }^{(2-5)}$, limitação da amplitude de movimento, e alterações posturais ${ }^{(6-8)}$. As alterações posturais, como aumento da lordose cervical e/ou lombar, aumento da cifose torácica e/ou alterações da posição da cabeça, podem gerar compensações na região cervical, pescoço e laringe ${ }^{(4,9)}$, contribuindo para a modificação do trato vocal e alteração da ressonância e a qualidade da $v^{(10-12)}$.

Um estudo retrospectivo(4) teve por objetivo observar características de tensão musculoesquelética em 260 sujeitos adultos submetidos a cirurgias de lesões benignas de pregas vocais (nódulos, pólipos e cistos), os autores compararam imagens dos sujeitos antes e após as cirurgias de laringe. Observaram que a tensão muscular esteve presente em ambos os grupos: pré e pós-tratamento cirúrgico. Os autores enfatizam a necessidade de tratamento complementar a terapia de voz, uma vez que a remoção das lesões, por meio de cirurgia, não melhorou o quadro de tensão musculoesquelética.

A relação entre a hipertonicidade dos músculos extrínsecos da laringe e a alteração da postura corporal foi avaliada previamente em 25 professoras com distúrbio de voz e os autores verificaram a clara relação existente entre a tensão muscular, alteração postural e disfonia ${ }^{(8)}$.

Outro estudo avaliou pacientes com disfonia e sujeitos sem alteração de voz, por meio da fotometria, e constatou que os 
disfônicos apresentaram assimetria de ombros, alteração no posicionamento da escápula e retificação com anteriorização da região cervical ${ }^{(9)}$. Com base nos resultados o autor ${ }^{(9)}$ concluiu que há correlação entre a disfonia e alteração postural.

Apesar dos trabalhos supracitados mostrarem que sujeitos com disfonia apresentam alteração postural, principalmente protrusão da cabeça, tensão dos músculos anteriores do pescoço e da região cervical, ambos avaliaram a postura e a disfunção muscular da região cervical de forma subjetiva. Alguns autores utilizaram a inspeção visual ${ }^{(7)}$ e a fotometria ${ }^{(8)}$ para constatar os desvios posturais. Assim sendo, não há na literatura estudos que analisam de forma objetiva a postura e a disfunção da região cervical em portadores de disfonia.

Para a avaliação objetiva das assimetrias e desvios posturais recomenda-se o uso da fotogrametria, método que possibilita o registro da inter relação entre diferentes partes do corpo e de alterações posturais sutis difíceis de serem mensuradas por outras formas de avaliação ${ }^{(13)}$. Além disso, alguns autores citam a alta confiabilidade da fotogrametria ${ }^{(14-18)}$, fato importante para a realização de pesquisas que envolvem avaliação postural ${ }^{(17)}$.

Como apresentado previamente, além de alterações posturais propriamente ditas, é muito comum a ocorrência de alterações funcionais da região cervical tais como: amplitude de movimento diminuída, dor nos músculos dessa região (podendo ocorrer no repouso e/ou durante a função e/ou durante a palpação) e condição articular alterada (ruídos articulares). Para avaliar a função da região crânio-cervical foi proposto o índice de disfunção crânio-cervical (IDCC), o qual tem como objetivo avaliar e acompanhar pacientes que apresentam disfunção crânio-cervical ${ }^{(19)}$.

Esse índice avalia a função cervical por meio da análise da amplitude de movimento, presença de dor durante o movimento, dor muscular e postura crânio-cervical e permite quantificar, de forma objetiva, as alterações funcionais existentes na região cervical $^{(19)}$, indicando assim a conduta terapêutica adequada.

Assim sendo, é importante enfatizar que a avaliação postural e funcional da região cervical são complementares, pois avaliação da postura mostra se há desalinhamento do segmento e a avaliação funcional mostra se o segmento avaliado apresenta algum déficit de função.

Com base na literatura consultada, a hipótese do presente estudo é que sujeitos disfônicos apresentam alteração postural da região crânio-cervical e por consequência alteração funcional da mesma região. Dessa forma, o objetivo do trabalho foi analisar a postura e a função da região crânio-cervical em sujeitos disfônicos.

\section{MÉTODOS}

\section{Amostra}

Participaram deste estudo 28 mulheres, caucasianas, na faixa etária de 20 a 44 anos (30,82 8 ,21 anos), divididas em grupo experimental e grupo controle. A pesquisa foi aprovada pelo Comitê de Ética em Pesquisa da Universidade Metodista de Piracicaba (UNIMEP), sob protocolo número 15/04.

O tamanho da amostra (N) foi determinado por meio do cálculo amostral com base nos valores de desvio padrão do ângulo de protrusão da cabeça. O cálculo amostral foi realizado utilizando-se o aplicativo GraphPad StatMate, versão 1.01i, 1998. Para intervalo de confiança de $95 \%$, power de $80 \%$ e $\alpha=0,05$ houve indicação de 12 voluntárias para o grupo controle e 16 voluntárias para o grupo experimental.

Fizeram parte do grupo experimental 16 mulheres (32,75 $\pm 8,01$ anos), com queixa de rouquidão, disfônicas, encaminhadas pelo Setor de Voz da Clínica Escola de Fonoaudiologia da UNIMEP. A análise perceptivo-auditiva fonoaudiológica classificou as vozes como rouco-soprosas de grau moderado, por meio da escala GRBASI $^{(20)}$. A avaliação laringológica confirmou a presença de nódulos bilaterais e fenda dupla à fonação (oito sujeitos) ou espessamento mucoso bilateral e fenda triangular médio-posterior à fonação (oito sujeitos). Optou-se por estudar apenas mulheres porque a incidência deste tipo de lesão apresenta-se maior nesse gênero, devido ao padrão feminino de proporção glótica ${ }^{(5,21,22)}$.

O grupo controle foi composto por 12 mulheres $(29,33 \pm 8,8$ anos) sem queixa de alteração vocal e com voz adaptada, constatada por análise perceptivo-auditiva fonoaudiológica.

Os dois grupos não apresentaram diferença quanto à idade $(\mathrm{p}=0,275)$.

Os critérios de exclusão para ambos os grupos foram considerados: prévio tratamento fonoaudiológico (para alteração vocal) e/ou fisioterapêutico (para correção postural). Tal critério foi adotado para evitar o risco da interferência de qualquer um dos tratamentos nos quadros de alterações vocais, alterações posturais e alterações funcionais da região cervical. Além disso, adotou-se como critério de exclusão sujeitos com alterações neurológicas e os que estavam em tratamento medicamentoso. Além disso, as voluntárias não deveriam apresentar idade superior a 50 anos, para excluir possíveis alterações decorrentes da idade, como processos degenerativos e não deveriam ser praticantes de atividade física, sendo todas sedentárias.

\section{Procedimentos}

A avaliação da postura da região cervical foi realizada por meio da fotogrametria e a avaliação da disfunção da região cervical pelo índice de disfunção crânio-cervical (IDCC) ${ }^{(19,23)}$.

Para tomada das fotos utilizou-se um simetrógrafo de fio (210 cm de altura, $95 \mathrm{~cm}$ de largura, com quadrados medindo 10 x $10 \mathrm{~cm}$ ) e na sua base foi colocado um posicionador de pés, o qual garantiu que todas as voluntárias estivessem posicionadas da mesma forma, em abdução de $10^{\circ}$ e rotação externa de $15^{\circ}$ da articulação coxo-femural.

$\mathrm{O}$ simetrógrafo foi posicionado à frente de uma parede pintada de azul causando efeito de fundo infinito, prevenindo a ocorrência de interferências.

Estes instrumentos permaneceram a $70 \mathrm{~cm}$ da parede e 110 cm da câmera fotográfica (Sony Cyber-shot $®, ~ 3,2$ megapixels) fixada sobre o tripé. A câmera fotográfica foi posicionada na linha central do simetrógrafo e alinhada em relação à voluntária, tendo o centro do processo xifóide como ponto de referência.

Cada voluntária foi instruída a permanecer na posição mais confortável possível e respirar normalmente, simulando a postura habitual. $\mathrm{O}$ examinador permaneceu sentado sempre 
na mesma cadeira posicionada atrás da câmera fotográfica na mesma linha de observação.

Para avaliar a protrusão da cabeça foi determinado, no plano sagital, o ângulo formado pela intersecção da reta que une o tragus da orelha e o processo espinhoso de $\mathrm{C}_{7}$ (sétima vértebra da coluna cervical) com a reta paralela ao solo. Optouse por analisar esse ângulo, pois esse apresenta repetibilidade aceitável, confiabilidade intra-examinadores excelente e interexaminadores muito bom ${ }^{(17)}$, sendo uma medida confiável para analisar a postura crânio-cervical.

O ângulo de protrusão da cabeça foi determinado apenas no plano sagital direito e sem flash devido a estudos pilotos realizados previamente, nos quais não foram observadas diferenças significativas entre os lados direito e esquerdo.

Para a análise das fotos foi utilizado o software Corel Draw 8.0, com o qual foi possível determinar o ângulo de protrusão da cabeça por meio da ferramenta dimensão. Para tal foram colocados marcadores de pele, estando um sobre o tragus da orelha e outro sobre o processo espinhoso de $\mathrm{C}_{7}$, uma reta foi traçada unindo esses dois marcadores e outra reta paralela ao solo. Na intersecção dessas retas foi formado um ângulo, o qual foi avaliado em ambos os grupos. Esta análise foi executada por três examinadores, os quais não eram responsáveis por fotografar as voluntárias, três vezes cada um, com um intervalo de uma semana para que os examinadores não memorizassem o valor encontrado.

Para fotogrametria as voluntárias foram orientadas a vestir traje de banho ou roupa íntima para melhor visibilidade das estruturas anatômicas da parte superior do tronco superior.

O IDCC permitiu a avaliação da região cervical. Esse índice avalia: a) amplitude de movimento (analisada por meio de goniometria da região cervical nos movimentos de flexão, extensão, inclinação para a direita e esquerda, e rotação para direita e esquerda); b) dor ao movimento cervical (avaliada durante os movimentos de flexão, extensão, rotação para a direita e esquerda e inclinação para a direita e esquerda); c) função da articulação cervical prejudicada (refere-se à presença de ruídos ou travamento durante a realização dos movimentos previamente descritos); d) dor muscular (graduada após palpação dos músculos esternocleidomastóideo, trapézio e paravertebrais, bilateralmente); e) medida da lordose cervical (a qual identifica se hiperlordose ou retificação cervical). Para cada um desses itens é possível atribuir três pontuações $(0,1$ ou 5 pontos), os quais são definidos de acordo com as respostas obtidas durante o exame físico.

Pela somatória dos pontos atribuídos em cada item de avaliado, é possível classificar o sujeito em quatro categorias, de acordo com a severidade da disfunção: sem disfunção (0 pontos), disfunção leve (1 a 4 pontos), disfunção moderada (5 a 9 pontos) e disfunção severa ( 10 a 25 pontos). Cabe esclarecer que a avaliação clínica, a pontuação e a análise do IDCC seguiram as recomendações de Wallace e Klineberg ${ }^{(19)}$.

O IDCC foi aplicado pelo pesquisador que realizou as fotos e foi analisado, apenas uma vez, por outro pesquisador que não conhecia a que grupo as voluntárias pertenciam.

Para possibilitar a análise estatística dos dados do IDCC foram atribuídos scores para classificação da disfunção crâniocervical (Quadro 1).
Quadro 1. Classificação e score atribuído para o índice de disfunção crânio-cervical

\begin{tabular}{|lc|}
\hline Classificação & Score atribuído \\
\hline Sem disfunção & 0 \\
Disfunção leve & 1 \\
Disfunção moderada & 2 \\
Disfunção severa & 3 \\
\hline
\end{tabular}

\section{Análise estatística}

Para a análise comparativa entre os grupos experimental e controle foi aplicado o teste de normalidade de Shapiro-Wilk utilizando o software JMP versão 3.1.6.2. Como os dados apresentaram distribuição normal foram analisados pelo teste t de Student utilizando o software Statistica.

Para testar a confiabilidade intra e inter-examinador da medida angular referente à posição da cabeça foi utilizado o índice de correlação intra-classe (ICC).

A correlação entre o índice de disfunção crânio-cervical e o ângulo de protrusão da cabeça foi realizada por meio do Coeficiente de Spearman.

Em todos os cálculos foi fixado um nível crítico de 5\% $(\mathrm{p}<0,05)$.

\section{RESULTADOS}

\section{Fotogrametria}

A análise dos valores do índice de correlação intra-classe mostrou confiabilidade excelente tanto intra como interexaminadores, pois os valores de ICC variaram de 0,97 a 1 .

Os resultados referentes à fotogrametria permitiram identificar que não houve diferença $(\mathrm{p}=0,2565)$ entre os valores médios do ângulo de protrusão da cabeça do grupo controle e do grupo experimental, indicando que a posição da cabeça no plano sagital não difere entre os grupos estudados, como mostra a Figura 1.

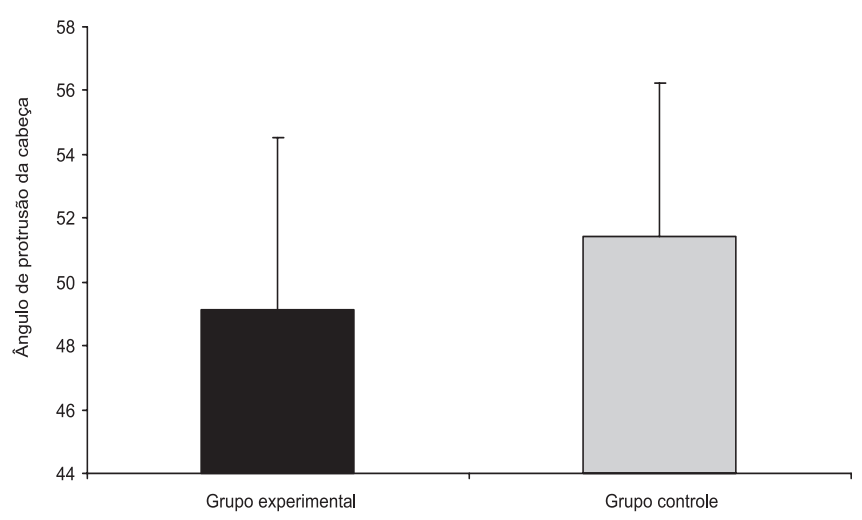

Teste t Student $(p=0,2565)$

Figura 1. Valores médios e desvio-padrão do ângulo de protrusão da cabeça no plano sagital, em graus, dos grupos experimental $(\mathrm{N}=16)$ e controle $(\mathrm{N}=12)$ 


\section{Índice de disfunção crânio-cervical}

Os resultados referentes ao IDCC permitiram identificar que houve diferença $(p=0,0053)$ entre o grupo controle e o grupo experimental (Tabela 1). Os resultados mostram que a maioria das mulheres do grupo experimental aprestou disfunção crânio-cervical moderada e severa.

Tabela 1. Grau de severidade da disfunção crânio-cervical nos grupos experimental e controle

\begin{tabular}{lcccc}
\hline Grupo & $\begin{array}{c}\text { Sem } \\
\text { disfunção }\end{array}$ & $\begin{array}{c}\text { Leve } \\
(\%)\end{array}$ & $\begin{array}{c}\text { Moderada } \\
(\%)\end{array}$ & $\begin{array}{c}\text { Severa } \\
(\%)\end{array}$ \\
\hline Experimental $(\mathrm{N}=6)$ & --- & 37,5 & 37,5 & 25 \\
Controle $(\mathrm{N}=12)$ & --- & 100 & --- & --- \\
\hline
\end{tabular}

Teste t Student $(\mathrm{p}=0,0053)$

\section{Correlação}

A análise da correlação entre o ângulo de protrusão da cabeça e o índice de disfunção crânio-cervical mostrou correlação não significativa ( $\mathrm{p}=0,39)$, como mostra a Figura 2.

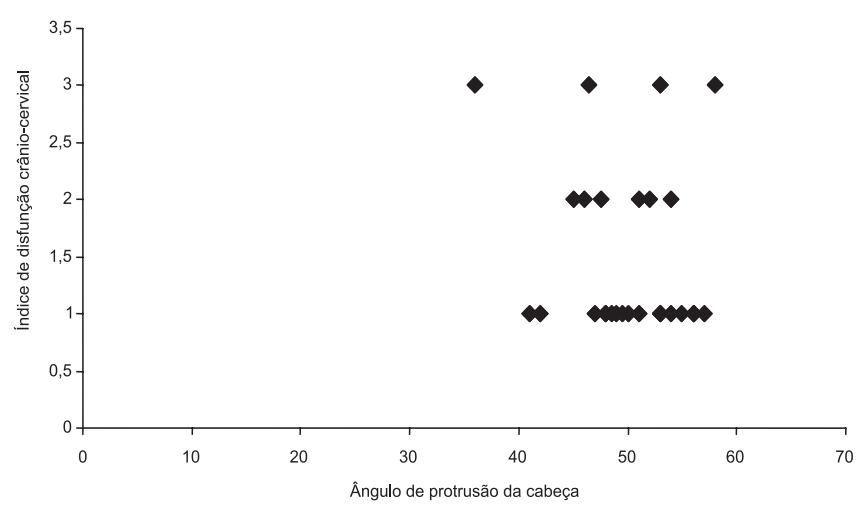

Coeficiente de Spearman $(p=0,39)$

Figura 2. Correlação entre os valores do ângulo de protrusão da cabeça e do índice de disfunção crânio-cervical (IDCC) (eixo y)

\section{DISCUSSÃO}

A disfunção crânio-cervical é uma condição comum na população. Estima-se que dois terços da população americana sofrerão dor cervical em algum momento da vida ${ }^{(24)}$. É caracterizada por dor cervical a qual pode ocorrer em repouso e/ou durante os movimentos da região cervical; limitação da amplitude dos movimentos da região; ruídos articulares e tensão dos músculos da região cervical, a qual pode ser avaliada pela presença de dor e/ou condição muscular durante a palpação.

Por meio do IDCC, no presente estudo, foi possível verificar que todas as mulheres disfônicas apresentaram disfunção crânio-cervical, sendo a disfunção modera e severa predominante. As mulheres do grupo controle também possuíam disfunção crânio-cervical, porém todas receberam classificação leve.

Para que a disfunção crânio-cervical seja diagnosticada, por meio do IDCC, o indivíduo pode apresentar um ou todos os sinais e sintomas descritos acima. Dessa forma, o que diferencia um indivíduo do outro é a severidade da disfunção.

Neste aspecto é importante ressaltar que a classificação leve, observada em todas as voluntárias do grupo controle, consiste na pontuação de 1 a 4 . Sendo assim, mulheres com dor à palpação no músculo trapézio, condição comum na maioria da população, já foram classificadas como portadoras de disfunção leve. Por outro lado, a maioria das mulheres do grupo experimental $(62,5 \%)$ foi classificada com disfunção crânio-cervical moderada a severa. Ou seja, possuíam mais alterações da amplitude de movimento cervical, dor ao movimento ou à palpação e função da articular prejudicada, quando comparados ao grupo controle.

Os resultados encontrados neste trabalho concordam com os achados da literatura, os quais revelam que a tensão dos músculos da região crânio-cervical está fortemente associada à disfonia, alterando inclusive a produção vocal ${ }^{(8,9,11)}$. Tal condição foi observada previamente em vinte e cinco professoras com disfonia ${ }^{(8)} \mathrm{e}$ os autores concluíram que, quanto maior o escore do índice tensão/postura maior é a alteração vocal e pior é a qualidade da voz. Da mesma forma, autores ${ }^{(4)}$ encontraram sinais de tensão musculoesquelética em sujeitos que foram submetidos à remoção de lesões benignas da laringe como nódulos, pólipos e cistos, concluindo que a tensão musculoesquelética é fator causal para quadros de disfonia por tensão muscular.

Em relação à avaliação da postura da região cervical em mulheres disfônicas, optou-se por utilizar a fotogrametria por ser um método confiável de avaliação postural. Há correlação positiva entre a avaliação da postura da cabeça por meio da fotogrametria e da radiografia, o que comprova a confiabilidade do método ${ }^{(25)}$. Além disso, o ângulo de protrusão da cabeça, utilizado no presente estudo, apresenta excelente confiabilidade intra-examinadores, e confiabilidade muito boa inter-examinadores ${ }^{(17)}$. Considerando tais afirmações, pode-se relatar que os dados do presente estudo corroboram achados prévios $^{(17)}$ em que, ao avaliar a confiabilidade do ângulo de protrusão da cabeça observou-se excelente confiabilidade intra e inter-examinadores.

As mulheres disfônicas, segundo os resultados do presente estudo, não mostraram alteração do ângulo de protrusão da cabeça quando comparadas às mulheres do grupo controle. Tais resultados não condizem com estudo prévio ${ }^{(9)}$ que comparou a postura corporal de 23 mulheres disfônicas com 20 mulheres sem alteração vocal e observou que as mulheres disfônicas apresentaram assimetria de ombros, alteração no posicionamento da escápula, retificação com anteriorização da região cervical. Acredita-se que a divergência entre os resultados encontrados ocorreu em função da metodologia utilizada, uma vez que o estudo supracitado não utilizou medida angular para quantificar as alterações posturais e no presente estudo a cintura escapular não foi avaliada pela fotogrametria.

De acordo com o presente estudo, pode-se relatar que o ângulo de protrusão da cabeça não difere entre mulheres disfônicas e não-disfônicas, não estando a disfonia diretamente relacionada à alteração da postura da região cervical. Entretanto, a disfunção crânio-cervical, índice importante para mensurar a função da região cervical, parece estar mais 
presente nas mulheres disfônicas do que a alteração da postura. Tornam-se necessários estudos objetivos que aprofundem esta relação, pois as observações clínicas na área da Fonoaudiologia sempre estiveram voltadas à postura relacionada à disfonia quando há a necessidade de se considerar os parâmetros de disfunção da região cervical.

Outro fator que merece ser ressaltado é que no presente trabalho a postura adotada pelas mulheres disfônicas durante a fala não foi avaliada. Acredita-se que possa existir alteração do padrão postural durante essa atividade, uma vez que estudos anteriores evidenciaram que esses sujeitos realizam esforço à fonação e possuem a caixa torácica constantemente elevada com movimentação restrita ${ }^{(3,4,21)}$.

Embora seja recomendada a avaliação postural durante a fala, pode-se sugerir que a disfonia está mais relacionada à disfunção da região crânio-cervical do que às alterações posturais dessa região. Dessa forma, o tratamento da disfonia deveria estar associado ao tratamento da disfunção crâniocervical, buscando diminuir a tensão muscular existente, bem como o restabelecimento da mobilidade normal desta região.

\section{CONCLUSÃO}

Nas condições experimentais realizadas, pode-se relatar que sujeitos disfônicos não apresentam alteração postural da região crânio-cervical. Porém, as mulheres disfônicas foram classificadas como portadoras de disfunção crânio-cervical mais acentuada que as clinicamente normais, o que sugere que as disfonias estejam mais relacionadas às alterações funcionais da região cervical do que às posturais da mesma região.

\section{AGRADECIMENTOS}

Ao Conselho Nacional de Desenvolvimento Científico e Tecnológico (CNPq) (processo n 475548/2003-5), ao Fundo de Apoio a Pesquisa da UNIMEP (FAP/UNIMEP), e ao Programa Institucional de Bolsas de Iniciação Científica (PIBIC/ $\mathrm{CNPq} / \mathrm{UNIMEP}$ ), pelo apoio concedido para realização dessa pesquisa.

\begin{abstract}
Purpose: To analyze the posture and the function of the craniocervical region in women with dysphonia. Methods: Twenty eight women participated in the study (31.25 \pm 8.14 years), divided into two groups: experimental ( $N=16$, patients with dyphonia) and control ( $\mathrm{N}=12$, clinically normal). The volunteers were submitted to evaluation of the Craniocervical Dysfunction Index (CDI) and photogrammetry, determining the anterior angle formed between the seventh cervical vertebra and the tragus, which corresponds to the head position in the sagittal plane. The analysis of the photos was carried out by three examiners, twice each, with an interval of one week between them. Data analysis used the Shapiro-Wilk test, followed by the Student's t-test, $(\mathrm{p}<0,05)$. Results: No difference was found between the mean values of the anterior angle of the control (50.92 \pm 5.18 degrees) and the experimental (49.63 \pm 5.46 degrees) groups in the photogrammetry $(\mathrm{p}=0.2565)$. The CDI showed that the experimental group had craniocervical dysfunction, which was mild in $37.5 \%$ of the cases, moderate, in $37.5 \%$, and severe in $25 \%$. In the control group, $100 \%$ of the volunteers had presented mild craniocervical dysfunction. Conclusion: There was no difference between the evaluated groups regarding head position. However, dysphonic women presented more severe craniocervical dysfunction than the group control.
\end{abstract}

Keywords: Posture/physiology; Dysphonia/complications; Spine; Head; Voice disorders; Photogrammetry

\section{REFERÊNCIAS}

1. Ferreira LP, Algodoal MJ, Andrada E, Silva MA. Avaliação da voz na visão (e no ouvido) do fonoaudiólogo: saber o que se procura para entender o que se acha. In: Marchesan IQ, Zorzi JL, Gomes ICD, organizadores. Tópicos em fonoaudiologia 1997/1998. São Paulo: Lovise; 1998. p. 393-413.

2. Behrman A. Common practices of voice therapists in the evaluation of patients. J Voice. 2005;19(3):454-69.

3. Anelli W, Xavier C. Novo enfoque de atendimento a pacientes disfônicos em instituição: grupos de orientação In: Marchesan IQ, Bolaffi C, Gomes ICD, Zorzi JL, organizadores. Tópicos em fonoaudiologia 1995. São Paulo: Lovise; 1995. p. 331-47.

4. Hsiung MW, Hsiao YC. The characteristic features of muscle tension dysphonia before and after surgery in benign lesions of the vocal fold. ORL J Otorhinolaryngol Relat Spec. 2004;66(5):246-54.

5. Imamura R, Domingos H. Disfonia espasmódica de adução, tremor vocal e disfonia de tensão muscular: é possível fazer o diagnóstico diferencial? Rev Bras Otorrinolaringol. 2006;72(4):434.
6. Angsuwarangsee T, Morrison M. Extrinsic laryngeal muscular tension in patients with voice disorders. J Voice. 2002;16(3):333-43.

7. Morrison MD, Rammage LA. Muscle misuse voice disorders: description and classification. Acta Otolaryngol. 1993;113(3): 428-34.

8. Kooijman PG, de Jong FI, Oudes MJ, Huinck W, van Acht H, Graamans $\mathrm{K}$. Muscular tension and body posture in relation to voice handicap and voice quality in teachers with persistent voice complaints. Folia Phoniatr Logop. 2005;57(3):134-47.

9. Nelli EA. Estudo da postura corporal em portadores da disfonia [tese na Internet]. Bauru: Hospital de Reabilitação de Anomalias Craniofaciais da Universidade de São Paulo; 2006. [citado 2007 Mai 31]. Disponível em: http://www.teses.usp.br/teses/disponiveis/61/61131/tde-14112006101425/.

10. Grini MN, Ouaknine M. Giovanni A. [Contemporary postural and segmental modification of forced voice]. Rev Laryngol Otol Rhinol (Bord).1998;119(4):253-7. French. 
11. Rubin JS, Blake E, Mathieson L. Musculoskeletal patterns in patients with voice disorders. J Voice. 2007;21(4):477-84.

12. Dromey C, Nissen SL, Roy N, Merrill RM. Articulatory changes following treatment of muscle tension dysphonia: preliminary acoustic evidence. J Speech Lang Hear Res. 2008;51(1):196-208.

13. Watson AW, Mac Donncha C. A reliable technique for the assessment of posture: assessment criteria for aspects of posture. J Sports Med Phys Fitness. 2000;40(3):260-70

14. Van Maanen CJ, Zonnenberg AJ, Elvers JW, Oostendorp RA. Intra/ interrater reliability of measurements on body posture photographs. Cranio. 1996;14(4):326-31. Erratum in: Cranio. 1997;15(1):8.

15. Hayes K, Walton JR, Szomor ZR, Murrel GA. Reliability of five methods for assessing shoulder range of motion. Aust J Physiother. 2001;47(4):289-94.

16. Sato TO, Vieira ER, Gil Coury HJC. Análise da confiabilidade de técnicas fotogramétricas para medir a flexão anterior do tronco. Rev Bras Fisioter. 2003;7(1):53-99.

17. Iunes DH, Castro FA, Salgado HS, Moura IC, Oliveira AS, BevilaquaGrossi D. Confiabilidade intra e interexaminadores e repetibilidade da avaliação postural pela fotogrametria. Rev Bras Fisiot. 2005;9(3):32734.
18. Sacco ICN, Aliberti S, Queiroz BWC, Pripas D, Kieling I, Kimura AA, et al. Confiabilidade da fotogrametria em relação a goniometria para avaliação postural de membros inferiores. Rev Bras Fisioter. 2007;11(5):411-7.

19. Wallace C, Klineberg IJ. Management of craniomandibular disorders. Part 1: A craniocervical dysfunction index. J Orofac Pain. 1993;7(1):838

20. Hirano M. Clinical examination of voice. New York: Springer-Verlag; c1981.

21. Behlau M. Voz: o livro do especialista. Rio de Janeiro: Revinter; c2001. Volume I.

22. Melo ECM, Brito LL, Brasil OCO, Behlau M, Melo DM. Incidência de lesões laríngeas não neoplásicas em pacientes com queixas vocais. Rev Bras Otorrinolaringol. 2001;67(6):788-94.

23. Wallace C, Klineberg IJ. Management of craniomandibular disorders. Part II: Clinical assessment of patients with craniocervical dysfunction. J Orofac Pain. 1994;8(1):42-54.

24. Côté P, Cassidy JD, Carroll L. The Saskatchewan Health and Back Pain Survey. The prevalence of neck pain and related disability in Saskatchewan adults. Spine (Phila Pa 1976). 1998;23(15):1689-98.

25. Visscher CM, De Boer W, Lobbezoo F, Habets LL, Naeije M. Is there a relationship between head posture and craniomandibular pain? J Oral Rehabil. 2002;29(11):1030-6. 